\title{
Motivo de afastamento do trabalho e qualidade de vida de servidores públicos municipais
}

\author{
Reason for absence from work and quality of life of municipal civil servants \\ Motivo de ausencia laboral y calidad de vida de los funcionarios municipales
}

Recebido: 04/01/2022 | Revisado: 09/01/2022 | Aceito: 11/01/2022| Publicado: 13/01/2022

\author{
Maylla Lienckvitz Barbosa \\ ORCID: https://orcid.org/0000-0002-7630-492X \\ Universidade de Franca, Brasil \\ E-mail: maylla-lienckvitz@hotmail.com \\ Silvio de Almeida Junior \\ ORCID: https://orcid.org/0000-0002-7949-4941 \\ Universidade de Franca, Brasil \\ E-mail: silvioalmeidajr@yahoo.com.br \\ Fabíola Pansani Maniglia \\ ORCID: https://orcid.org/0000-0002-3281-9470 \\ Universidade de Franca, Brasil \\ E-mail: fabiola.maniglia@unifran.edu.br \\ Salvador Boccaletti Ramos \\ ORCID: https://orcid.org/0000-0001-5422-1309 \\ Universidade de Franca, Brasil \\ E-mail: salvador.ramos@unifran.edu.br
}

\begin{abstract}
Resumo
Este estudo identificou os motivos de afastamento do trabalho e a relação com as características sociodemográficas, laborais e de qualidade de vida (QV) de servidores públicos de um município de Minas Gerais a partir de um estudo observacional, transversal e quantitativo, realizado com 70 servidores públicos, por meio de questionário WHOQOLBREF. Os resultados apontaram que 64\% dos servidores já se afastaram do trabalho. O motivo de afastamento mais ocorrente foi a depressão $(p<0,01)$. Houve diferença com relação ao afastamento entre homens e mulheres $(p<0,05)$. Não houve diferença nos resultados de QV entre os grupos afastados e não afastados. Sugere-se a implementação de ações de promoção da saúde mental entre os servidores para a melhoria na QV e diminuição da necessidade de se ausentar do trabalho.
\end{abstract}

Palavras-chave: Depressão; Promoção da saúde; Risco ocupacional; Serviço social; Qualidade de vida.

\begin{abstract}
This study identified the reasons for absence from work and the relationship with the sociodemographic, labor and quality of life (QL) characteristics of public servants in a municipality in Minas Gerais, based on an observational, cross-sectional and quantitative study carried out with 70 servants. through the WHOQOL-BREF questionnaire. The results showed that $64 \%$ of the servers have already left work. The most frequent reason for leaving was depression $(p<0.01)$. There was a difference in terms of leave between men and women $(p<0.05)$. There was no difference in QoL results between the distant and non-retired groups. The implementation of mental health promotion actions among servers is suggested to improve QOL and reduce the need to be absent from work.
\end{abstract}

Keywords: Depression; Health promotion; Occupational risk; Social service; Quality of life.

\section{Resumen}

Este estudio identificó los motivos de ausencia laboral y la relación con las características sociodemográficas, laborales y de calidad de vida (CV) de los servidores públicos de un municipio de Minas Gerais, a partir de un estudio observacional, transversal y cuantitativo realizado con 70 servidores a través del cuestionario WHOQOL-BREF. Los resultados mostraron que el $64 \%$ de los servidores ya han salido del trabajo. El motivo de salida más frecuente fue la depresión ( $\mathrm{p}<0,01)$. Hubo una diferencia en términos de licencia entre hombres y mujeres $(\mathrm{p}<0.05)$. No hubo diferencia en los resultados de la calidad de vida entre los grupos distantes y no jubilados. Se sugiere la implementación de acciones de promoción de la salud mental entre servidores para mejorar la calidad de vida y reducir la necesidad de ausentarse del trabajo.

Palabras clave: Depresión; Promoción de la salud; Riesgo laboral; Servicio social; Calidad de vida. 


\section{Introdução}

O trabalho é uma das atividades mais antigas dentro da sociedade, demonstrando a necessidade e efetividade da sua extensão na construção de comunidades produtivas e prósperas, fazendo com que o trabalhador molde seu estilo de vida e ações sociais a partir de lucros obtidos por tal atividade (Chaves, 2020; Tavares, 2020). Dessa forma, podemos entender que a atividade laboral exercida pelo indivíduo, tem relação intima com a qualidade de vida deste. A partir desta definição, defendese a importância de se contemplar os domínios físico, psicológico, social e ambiental para avaliar a qualidade de vida (Guimarães et al., 2020; Lirio et al., 2020).

Ao considerar essa perspectiva abrangente, diversos autores têm investigado sobre a qualidade de vida de trabalhadores, uma vez que os indivíduos passam uma significativa parte de suas vidas desempenhando as atividades laborais e estas podem influenciar em todos os domínios supracitados (de Oliveira Meller et al., 2020; Costa et al., 2012).

O trabalho é descrito como atividade essencial ao homem, que apresenta relação com aspectos pessoais e sociais frente a sociedade, contudo, quando o trabalhador é acometido por alguma doença, o afastamento do trabalho pode ser necessário (Santi et al., 2018; Tolfo \& Piccinini, 2007). Afastamento do ambiente laboral como absenteísmo, destaca que a ausência, decorrente de uma incapacidade do indivíduo, afeta a continuidade de atividades e onera os cofres públicos, tanto pela não produtividade, mas também pelas despesas relacionadas à reabilitação do servidor (Junior et al., 2020; Santi et al., 2018).

Estudos indicam que o aumento das doenças na classe trabalhadora esteja associado às exaustivas jornadas de trabalho e consequente diminuição do tempo para alimentação, prática regular de exercício físico e lazer com a família, que somados à sobrecarga emocional e mental, repercutem negativamente na sua qualidade de vida e levam ao aumento das taxas de afastamento do trabalho (Marinho \& Vieira, 2019).

Acredita-se, portanto, que entender a dimensão do problema dos afastamentos do trabalho implica inicialmente em conhecer o registro dos mesmos e então, levantar os motivos que estão levando à piora da qualidade de vida dos trabalhadores. Desta forma, o presente estudo teve como objetivo identificar os motivos de afastamento do trabalho e a relação com as características sociodemográficas, laborais e de qualidade de vida de servidores públicos de um município de Minas Gerais.

\section{Metodologia}

Trata-se de um estudo transversal, observacional e quantitativo, realizado com servidores da Secretaria de Saúde de um município de Minas Gerais, visando a identificação dos motivos de afastamento do trabalho e da qualidade de vida dos servidores públicos de uma cidade do estado de Minas Gerais. A pesquisa foi aprovada pelo Comitê de Ética em Pesquisa da Universidade de Franca sob o parecer número 3.558.386.

Trata-se de uma pesquisa realizada com servidores públicos da Secretaria Municipal de Saúde (SMS) de um município de Minas Gerais. Optou-se por esta secretaria, por se tratar de uma das maiores dentro do município em número de funcionários, e pela sua relação direta e envolvimento com a área da saúde. A Secretaria Municipal de Saúde em questão tem como atribuições: planejar, desenvolver, orientar, coordenar e executar a política de saúde do município, compreendendo tanto o cuidado ambulatorial quanto o hospitalar, sendo também de nossa responsabilidade planejar, desenvolver e executar as ações de vigilância sanitária e epidemiológica afetas à nossa competência e em consonância com os princípios do SUS. A esta Secretaria também compete, buscar excelência nas ações direcionadas a integridade na saúde e na qualidade de vida dos cidadãos em concordância com a Lei Orgânica da Saúde (Lei 8080/90 e 8142/90), que apresenta como princípios e diretrizes a universalidade do acesso aos serviços de saúde, a integralidade da assistência e a igualdade da assistência à saúde.

A primeira etapa da coleta de dados da pesquisa consistiu no convite realizado com antecedência pelo responsável do setor para a participação da pesquisa. Para tanto foram adotados como critério de inclusão no estudo: servidores ativos, 
maiores de 18 anos, de ambos os sexos. Foram excluídos aqueles servidores com deficiência visual que incapacitava a leitura do questionário.

De um total de 95 servidores, 70 preencheram os critérios de inclusão e aceitaram participar voluntariamente do estudo, assinando o Termo de Consentimento Livre e Esclarecido (TCLE). Para fins de facilitar a execução da pesquisa os 70 indivíduos recrutados foram divididos em 5 grupos de 14 pessoas e cada grupo compareceu a um encontro pré-agendado para a coleta dos dados.

As informações sociodemográficas e a respeito do afastamento do trabalho, presença de doenças crônicas e uso medicamento foram colhidas por meio de um questionário semiestruturado e para a avaliação da qualidade de vida foi utilizado o questionário WHOQOL-BREF, no qual é composto por 26 questões, com 24 facetas relacionadas à qualidade de vida e agrupadas em quatro domínios: saúde física, saúde psicológica, relações sociais e meio ambiente.

Após a coleta, os dados foram submetidos às análises. Para a análise descritiva, obteve-se os valores absolutos e relativos e de média aritmética, desvio-padrão, mínimo e máximo. A análise estatística das variáveis categóricas foi realizada por meio do teste Qui-quadrado e da regressão logística. Já para se obter a comparação das médias dos escores total e por domínio para afastados e não afastados, no que se refere à qualidade vida, bem como a comparação das médias dos escores total e por domínio para depressivos e não depressivos, utilizou-se o Teste-t e a estatística “d” de Cohen (Ferguson, 2009). Para todas as análises utilizou-se o software jamovi v1.1 (2019) e foi adotado o nível de significância de 5\% (p<0,05).

\section{Resultados e Discussão}

Foram entrevistados 70 servidores públicos municipais que atuavam na Atenção Básica de Saúde, dos quais $64 \%$ já haviam se afastado do trabalho. A média da idade dos participantes da pesquisa foi de $47 \pm 10,1$ anos e as demais informações sobre a caracterização dos mesmos se encontram na Tabela 1.

Tabela 1 - Características sociodemográficas dos participantes do estudo (n = 70). Uberaba (MG), 2019.

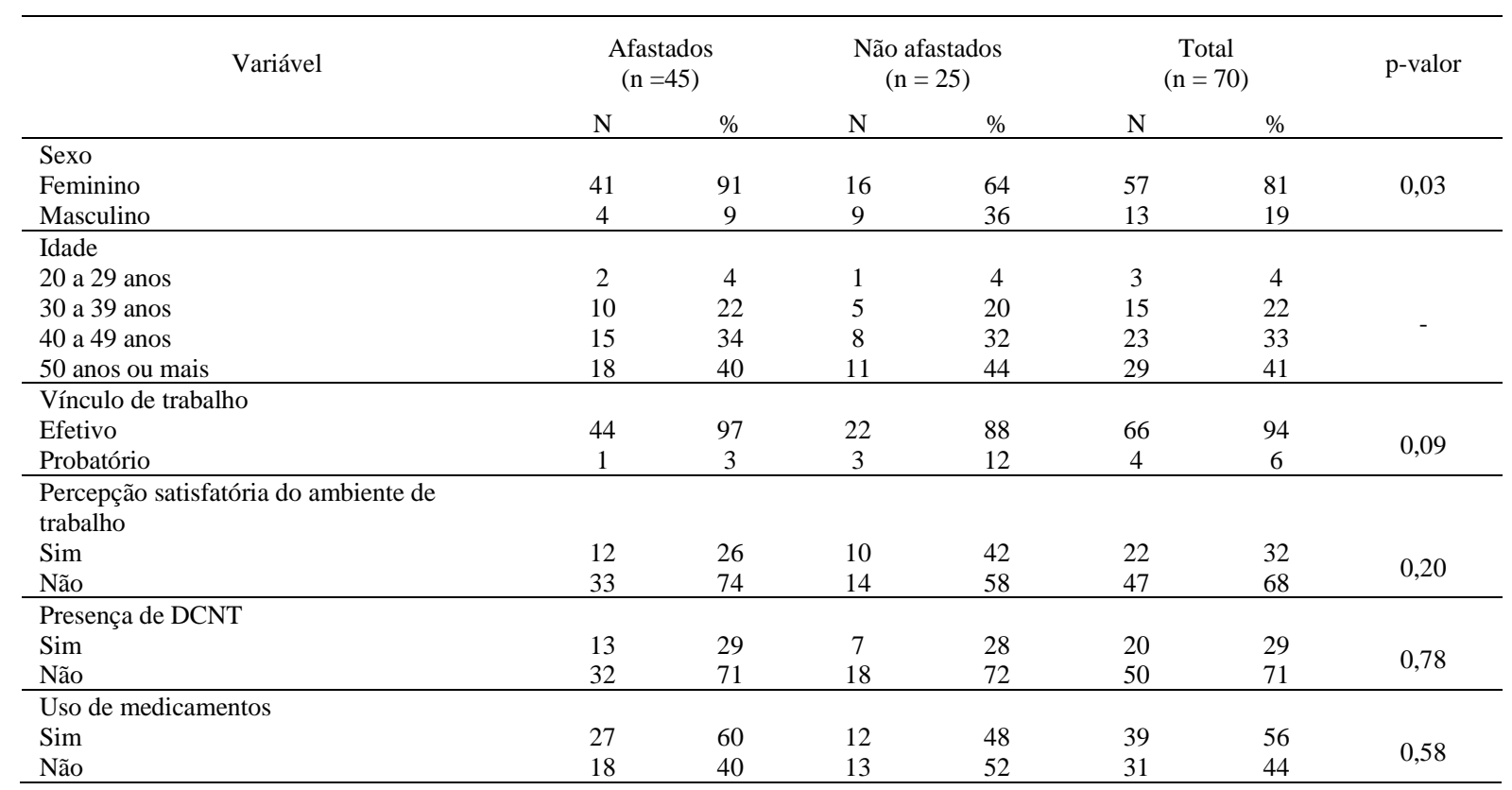

DCNT: Doenças Crônicas não Transmissíveis. Fonte: Dados dos autores (2022).

A respeito das características sociodemográficas da amostra da presente pesquisa, verificou-se que as mulheres do presente estudo se afastaram mais do que os homens $(\mathrm{p}<0,05)$. A sobrecarga de papéis desempenhados pelas mulheres exerce 
forte influência em sua saúde, em que, muitas vezes essas mulheres trabalham em tempo integral e, ao chegarem em casa, é preciso empreender tempo aos papéis familiares. Essa dupla jornada, vem se caracterizando em uma tarefa complexa, podendo comprometer a sua saúde física e mental das mulheres (Junior et al., 2020; Baasch et al., 2017; Natal \& Faiman, 2010).

Quanto à percepção sobre o ambiente de trabalho, esta não esteve associada com os afastamentos, assim como a presença de Doenças Crônicas não Transmissíveis (DCNT) e o uso de medicamentos. Pesquisadores apontam que o ambiente de trabalho desfavorável colabora para o adoecimento e consequente afastamento do trabalho (Antunes \& Praun, 2015), no entanto, no presente estudo os afastamentos não foram ocasionados pelas condições laborais.

Vale ressaltar que o ambiente de trabalho deve ser entendido também quanto às relações que acontecem neste cenário, as quais podem sofrer precarizações de diversos aspectos e que talvez não foram consideradas pelos trabalhadores (Granadeiro et al., 2020; Franco et al., 2010). Deve-se ainda lembrar que a pesquisa foi aplicada no ambiente de trabalho e que talvez algum servidor possa ter se sentir constrangido ou inseguro em relatar honestamente a própria opinião.

Pesquisas realizadas com servidores públicos mostram que, ainda que o servidor público conte com certa estabilidade no trabalho, ou seja, o risco de demissão é menor quando comparado ao setor privado, o servidor conta com outros elementos que podem afetar a sua saúde, tais como: precarização do trabalho, a degradação do ambiente de trabalho, as responsabilizações pelas deficiências dos serviços, a instabilidade motivadas pelas mudanças políticas, às ações descontinuadas, o acúmulo de funções, além do estereótipo de morosidade (Baasch et al., 2017; Nunes \& Lins, 2009).

A respeito da não associação entre os afastamentos e as DCNT, uma das possíveis justificativas, é que estas doenças, apesar de serem um problema crescente e de considerável magnitude, são silenciosas e não provocam dor ou incapacidade imediata e, possivelmente, por essa razão, não estiveram associadas ao afastamento do ambiente de trabalho no presente estudo (Malta et al., 2015). A Figura 1 apresenta os motivos dos afastamentos entre os trabalhadores.

Figura 1 - Motivo dos afastamentos na população do estudo (n=45). Uberaba (MG), 2019.

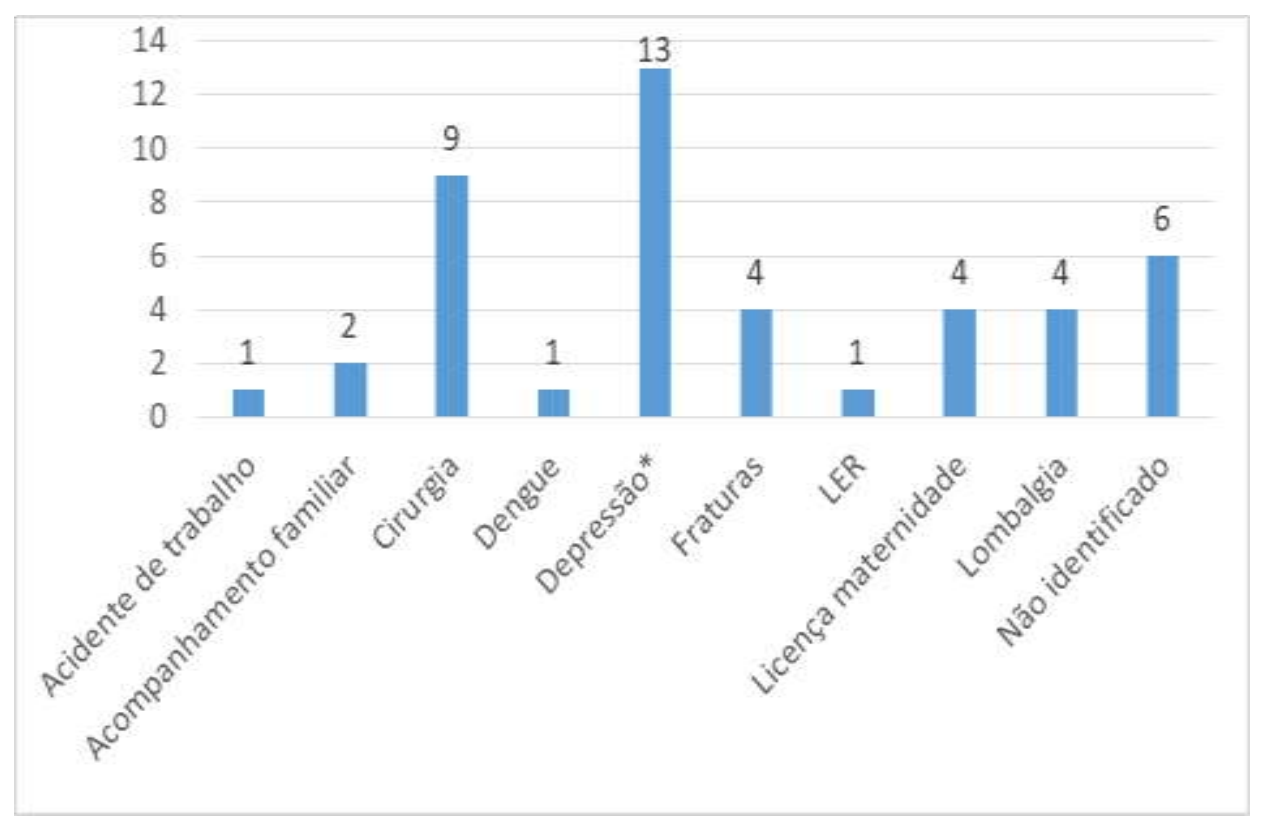

Fonte: Dados dos autores (2022).

A respeito dos motivos que levaram ao afastamento, observou-se que o mais ocorrente foi a depressão e os problemas associados, como: síndrome do pânico, esgotamento e ansiedade. A análise estatística mostrou uma associação positiva entre a ocorrência de depressão e os afastamentos $(\mathrm{p}<0,01)$ e deve-se considerar que dentre os indivíduos que utilizavam medicamentos, $28 \%$ faziam uso para tratamento psiquiátrico. 
Malta et al. (2015), descrevem a depressão como um transtorno mental afetivo caracterizado, principalmente, por oscilações de humor, redução da energia e, consequentemente, diminuição das atividades. $\mathrm{O}$ afastamento devido problemas neuropsiquiátricos, incluindo ansiedade, depressão ou outros quadros correlatos, são amplamente abordados entro da literatura, visto que a produção econômica estimada em milhões de dólares, sendo apontada como doença de maior crescimento entre os casos de afastamento entre o mundo (Feitosa \& Fernandes, 2020; Almeida Junior et al., 2019). É visto que a maior incidência de ocorrências de afastamento por depressão, são em mulheres, correlacionando com o que é encontrado na literatura, em que mulheres tem três vezes mais chances de desenvolverem quadros depressivos (Feitosa \& Fernandes, 2020; Malta et al., 2015).

Uma pesquisa realizada por Bastos et al., (2018) com servidores públicos confirmou os achados do presente estudo e corroborou com as evidências nacionais e mundiais. De acordo com os pesquisadores os transtornos mentais e comportamentais representaram a principal causa de afastamento, durante o ano da pesquisa, correspondendo a $23 \%$ do total de afastamentos por tratamento de saúde. Para os autores, as ausências no trabalho por motivo de transtornos mentais têm apresentado importante visibilidade nos últimos anos, acometendo um número cada vez maior de trabalhadores no Brasil (Bastos et al., 2018; Almeida Junior et al., 2019).

Sabe-se que as incapacidades e limitações impostas pelas doenças podem afetar significativamente a QV. A Tabela 2 apresenta a pontuação média total e por domínios do questionário de QV entre os indivíduos afastados e não afastados.

Tabela 2 - Resultados da avaliação da Qualidade de Vida entre os grupos afastados e não afastados $(\mathrm{n}=70)$. Uberaba $(\mathrm{MG})$, 2019.

\begin{tabular}{lcccc}
\hline \multirow{2}{*}{ Qualidade de Vida por dimensão } & \multicolumn{2}{c}{$\begin{array}{c}\text { Afastados } \\
(\mathrm{n}=45)\end{array}$} & \multicolumn{2}{c}{$\begin{array}{c}\text { Não afastados } \\
(\mathrm{n}=25)\end{array}$} \\
\hline Total & Média & DP & Média & DP \\
Qualidade de Vida Geral & 84,1 & 13,7 & 89,1 & 11,2 \\
Domínio Físico & 6,6 & 1,7 & 7,3 & 1,7 \\
Domínio Psicológico & 28,0 & 5,3 & 29,5 & 3,5 \\
Domínio Meio Ambiente & 15,1 & 3,5 & 16,6 & 2,8 \\
Domínio Relações Sociais & 20,0 & 3,3 & 21,1 & 2,8 \\
\hline
\end{tabular}

DP: desvio-padrão. Fonte: Dados dos autores (2022).

No que se refere à $\mathrm{QV}$, não houve diferença entre os grupos afastados e não afastados quanto ao resultado da soma das pontuações dos domínios da QV, bem como da pontuação total. No entanto, quando estes mesmos resultados foram comparados entre indivíduos afastados por depressão e por outros motivos, verificou-se uma diferença estatisticamente significativa para a pontuação total $(p=0,02$; Cohen's $d=0,75)$ e para o domínio psicológico $(p=0,04$; Cohen's $d=0,67)$. O fator depressão é descrito como inversamente proporcional ao índice de qualidade de vida, uma vez que quanto mais sintomas de transtornos depressivos o paciente apresenta, menor a pontuação total da QV e principalmente sobre o domínio psicológico (Castro et al., 2018).

Vale mencionar algumas limitações do presente estudo. Uma vez que seu recorte foi transversal, os indivíduos foram avaliados somente em um momento. Entende-se, portanto, que o resultado da QV, obtido pelo questionário, poderia refletir alguma condição específica daquele momento, sendo ela positiva ou não. Acredita-se então que estudos sistemáticos e longitudinais devam ser incentivados para aumentarem a confiabilidade dos resultados e possibilitarem o desenvolvimento de ações de promoção da saúde para estes trabalhadores. 


\section{Conclusão}

Houve um elevado número de afastamentos do trabalho entre os servidores públicos do município de Minas Gerais, especialmente no público feminino. Os motivos dos afastamentos não estiveram associados a doenças crônicas orgânicas, mas sim à depressão, a qual demonstrou impacto negativo no domínio psicológico da QV, entre os afastados. Sugere-se a continuidade das investigações sobre os afastamentos e os fatores relacionados para a implementação de ações de promoção da saúde que colaborem para o tratamento do estado depressivo, bem como para a sua prevenção, entre os servidores. Acredita-se que desta forma a qualidade de vida dos trabalhadores melhorará e repercutirá na diminuição da necessidade de se ausentar do trabalho.

\section{Referências}

Almeida Junior, S. de, Silva, M. M. da, Popolim, R. S., Gonçalves, C. R., Melo, M. R. S. de, \& Bulgo, D. C. (2019). Dissemination of knowledge and scientific production in professionalizing courses: A report of experience. Pubsaúde, 2, 1-8. https://doi.org/10.31533/pubsaude2.a008

Antunes, R., \& Praun, L. (2015). A sociedade dos adoecimentos no trabalho. Serviço Social \& Sociedade, 407-427. https://doi.org/10.1590/0101-6628.030

Baasch, D., Trevisan, R. L., \& Cruz, R. M. (2017). Perfil epidemiológico dos servidores públicos catarinenses afastados do trabalho por transtornos mentais de 2010 a 2013. Ciência \& Saúde Coletiva, 22, 1641-1650. https://doi.org/10.1590/1413-81232017225.10562015

Bastos, M. L. A., Junior, G. B. da S., Domingos, E. T. C., Araújo, R. M. O. de, \& Santos, A. L. dos. (2018). Afastamentos do trabalho por transtornos mentais: Um estudo de caso com servidores públicos em uma instituição de ensino no Ceará, Brasil. Revista Brasileira de Medicina do Trabalho, 16(1), 53-59. https://doi.org/10.5327/Z1679443520180167

Castro, A. Q. de, Junior, P. R. F., Filoni, E., Alves, V. L. dos S., \& Jorge, V. C. (2018). Avaliação da correlação entre depressão e o índice de qualidade de vida em indivíduos com lombalgia. Life Style, 5(2), 31-43. https://doi.org/10.19141/2237-3756.lifestyle.v5.n2.p31-43

Chaves, A. B. P. (2020). Da Planta taylorista/fordista ao capitalismo de plataforma: As engrenagens da exploração do trabalho. Research, Society and Development, 9(6), e01963473-e01963473. https://doi.org/10.33448/rsd-v9i6.3473

Costa, C. S. N. da, Freitas, E. G. de, Mendonça, L. C. de S., Alem, M. E. R., \& Coury, H. J. C. G. (2012). Capacidade para o trabalho e qualidade de vida de trabalhadores industriais. Ciência \& Saúde Coletiva, 17(6), 1635-1642. https://doi.org/10.1590/S1413-81232012000600026

de Oliveira Meller, F., Grande, A. J., Quadra, M. R., Doyenart, R., \& Schäfer, A. A. (2020). Qualidade de vida e fatores associados em trabalhadores de uma Universidade do Sul de Santa Catarina. Cadernos Saúde Coletiva, 28, 87-97. https://doi.org/10.1590/1414-462X202028010327

Feitosa, C. D. A., \& Fernandes, M. A. (2020). Afastamentos laborais por depressão. Revista Latino-Americana de Enfermagem, 28. https://doi.org/10.1590/1518-8345.3634.3274

Ferguson, C. J. (2009). An effect size primer: A guide for clinicians and researchers. Professional Psychology: Research and Practice, 40(5), 532-538. https://doi.org/10.1037/a0015808

Franco, T., Druck, G., \& Seligmann-Silva, E. (2010). As novas relações de trabalho, o desgaste mental do trabalhador e os transtornos mentais no trabalho precarizado. Revista Brasileira de Saúde Ocupacional, 35, 229-248. https://doi.org/10.1590/S0303-76572010000200006

Granadeiro, D. da S., Oliveira, E. B., Passos, J. P., Marques, F. C., Senna, N. V. M., \& Higa, G. J. O. (2020). Precarização do trabalho em serviço de atendimento móvel de urgência e a saúde do trabalhador. Research, Society and Development, 9(2), e181922032-e181922032. https://doi.org/10.33448/rsdv9i2.2032

Guimarães, M. B., Nunes, J. A., Velloso, M., Bezerra, A., Sousa, I. M. de, Guimarães, M. B., Nunes, J. A., Velloso, M., Bezerra, A., \& Sousa, I. M. de. (2020). As práticas integrativas e complementares no campo da saúde: Para uma descolonização dos saberes e práticas. Saúde e Sociedade, 29(1). https://doi.org/10.1590/s0104-12902020190297

Junior, S. de A., Cardoso-Brito, V., Moreira, M. E. S., Melo, M. R. S. de, Andrade, G., \& Bulgo, D. C. (2020). Biosafety evaluation and characterization of occupational risks in a ready care unit paulista, Brazil. Research, Society and Development, 9(2), 74922028. https://doi.org/10.33448/rsd-v9i2.2028

Lirio, A. B., Gallon, S., Costa, C., \& Pauli, J. (2020). PERCEPÇÕES DA QUALIDADE DE VIDA NO TRABALHO NAS DIFERENTES GERAÇÕES. Gestão \& Regionalidade, 36(107). https://doi.org/10.13037/gr.vol36n107.5398

Malta, D. C., Stopa, S. R., Szwarcwald, C. L., Gomes, N. L., Silva, J. B., \& Reis, A. A. C. dos. (2015). A vigilância e o monitoramento das principais doenças crônicas não transmissíveis no Brasil—Pesquisa Nacional de Saúde, 2013. Revista Brasileira de Epidemiologia, 18, 03-16. https://doi.org/10.1590/19805497201500060002

Marinho, M. O., \& Vieira, F. de O. (2019). A jornada exaustiva e a escravidão contemporânea. Cadernos EBAPE.BR, 17(2), 351-361.

Natal, T., \& Faiman, C. J. S. (2010). Repercussões do afastamento do trabalho na identidade de homens e mulheres - um estudo comparativo. Saúde, Ética \& Justiça (e-ISSN 2317-2770), 15(1), 16-27. https://doi.org/10.11606/issn.2317-2770.v15i1p16-27 
Research, Society and Development, v. 11, n. 1, e50411125367, 2022

(CC BY 4.0) | ISSN 2525-3409 | DOI: http://dx.doi.org/10.33448/rsd-v11i1.25367

Nunes, A. V. de L., \& Lins, S. L. B. (2009). Servidores públicos federais: Uma análise do prazer e sofrimento no trabalho. Revista Psicologia Organizações e Trabalho, 9(1), 51-67.

Santi, D. B., Barbieri, A. R., \& Cheade, M. de F. M. (2018). Absenteísmo-doença no serviço público brasileiro: Uma revisão integrativa da literatura. Revista Brasileira de Medicina do Trabalho, 16(1), 71-81. https://doi.org/10.5327/Z1679443520180084

Tavares, M. A. (2020). Envelhecimento e trabalho na sociedade capitalista. Revista Katálysis, 23, 143-151. https://doi.org/10.1590/1982-02592020v23n1p143 Tolfo, S. da R., \& Piccinini, V. (2007). Sentidos e significados do trabalho: Explorando conceitos, variáveis e estudos empíricos brasileiros. Psicologia \& Sociedade, 19, 38-46. https://doi.org/10.1590/S0102-71822007000400007 